\title{
THE
}

1975

\section{Scaling behavior at zero-temperature critical points}

George A. Baker Jr.

Jill C. Bonner

University of Rhode Island

Follow this and additional works at: https://digitalcommons.uri.edu/phys_facpubs

Terms of Use

All rights reserved under copyright.

\section{Citation/Publisher Attribution}

Baker, Jr., G. A., \& Bonner, J. C. (1975). Scaling behavior at zero-temperature critical points. Phys. Rev. B, 12(9), 3741-3744. doi: 10.1103/PhysRevB.12.3741

Available at: http://dx.doi.org/10.1103/PhysRevB.12.3741

This Article is brought to you for free and open access by the Physics at DigitalCommons@URI. It has been accepted for inclusion in Physics Faculty Publications by an authorized administrator of DigitalCommons@URI. For more information, please contact digitalcommons-group@uri.edu. 


\title{
Scaling behavior at zero-temperature critical points*
}

\author{
George A. Baker, Jr. and Jill C. Bonner \\ Applied Mathematics Department, Brookhaven National Laboratory, Upton, New York 11973
}

(Received 3 June 1975)

\begin{abstract}
A scaling form for the logarithm of the partition function suitable for a zero-temperature critical point is obtained and found to hold for the spherical model in less than two dimensions and the classical $n$-component Heisenberg linear chain. Nevertheless, several cases are found where the critical-exponent relations involving the specific heat fail. These anomalous cases do not imply a breakdown of the scaling implicit in the basic formulation of renormalization-group theory.
\end{abstract}

\section{INTRODUCTION}

The renormalization-group approach ${ }^{1}$ to the problem of critical phenomena depends explicitly on an assumption which requires a certain scaling form for the logarithm of the partition function. In this paper we investigate the spherical model and the $n$-component classical Heisehberg model for those spatial dimensions for which the critical temperature goes to zero. We find in the case of the linear three-component classical Heisenberg model and also the linear spin- $\frac{1}{2}$ Ising model that anomalies do occur. These anomalies imply the failure of the expected scaling relations among the critical indices which involve $\alpha$, the specific-heat index. However, this failure does not invalidate the scaling form assumed by the renormalization-group approach, as one might perhaps initially have feared, but reflects certain special situations.

Hyperscaling for a zero-temperature critical point implies that

$$
\lim _{T \rightarrow 0}\left(\frac{\ln \left(C_{H} \xi^{d}\right)}{-\ln T}\right)=0
$$

using the usual definition of critical exponents. Here $\xi$ is the range of correlation, $C_{H}$ is the speciic heat at constant magnetic field, $d$ is the spatial dimension, and $T$ is the absolute temperature. Specifically, the anomalies that occur are that instead of zero for the right-hand side of Eq. (1), we obtain 2 for the one-dimensional Ising model and 1 for the classical Heisenberg chain. If the "singular part" of the specific heat is used instead of the dominant part, the result, $-\infty$, is obtained instead for the linear classical Heisenberg model!

\section{REFORMULATED SCALING AND THE RENORMALIZATION GROUP}

Wilson ${ }^{2}$ explicitly assumes that there exists a renormalization-group transformation with a fixed point and a particular, simple form near the fixed point. He uses this assumption to show that

$$
\begin{aligned}
& \Gamma\left(\overrightarrow{\mathrm{q}}_{1}, \overrightarrow{\mathrm{q}}_{2}, \ldots, \overrightarrow{\mathrm{q}}_{n} ; T\right) \\
& \quad=\xi^{(n-1) d-n d} s_{A}^{n} F\left(\xi \overrightarrow{\mathrm{q}}_{1}, \xi \overrightarrow{\mathrm{q}}_{2}, \ldots, \xi \overrightarrow{\mathrm{q}}_{n}\right),
\end{aligned}
$$

where $\zeta_{A}$ is a normalization factor, $F$ is a function assumed not to vanish (but it can in anomalous cases), and the $\Gamma$ 's are the Fourier transforms of the truncated spin-spin correlation functions. By truncated correlation functions, we mean the Ursell functions, ${ }^{3}$ or, in the language of statistics, ${ }^{4}$ the cumulants, instead of the moments (correlation functions). The rest of Wilson's renormalization-group derivations appear to depend in an essential way on this result.

As far as the thermodynamics of the system is concerned, we need to know the logarithm of the partition function

$$
Z=\operatorname{Tr}[\exp (-\beta \mathcal{H C}+\beta m H \mathscr{T})],
$$

where $\beta=1 / k T, \mathfrak{H}$ is the Hamiltonian, $H$ is the magnetic field, $\mathfrak{T}$ is the magnetization variable, and $m$ is the magnetic moment per spin. The form of $(\ln Z) / N$, where $N$ is the number of spins, can be deduced from (2) by setting $\overrightarrow{\mathrm{q}}_{i}=0$. We then conclude

$$
(\ln Z) / N \simeq \xi^{-d} Y\left(H \xi^{d-d} s\right),
$$

where $d_{s}$ is called the anomalous dimension of the spin field. The function $Y$ is the formal sum of a power series in $H^{2}$ whose coefficients are derived from (2). This form is supposed to hold at least for $T \gtrsim T_{c}$, and $H \ll \xi^{d} s^{-d}$.

In order to extend the form (4) to the case of zero critical temperature, it is helpful to remember the various definitions for the zero-field susceptibility, specific heat, spin-spin correlation functions, correlation length, and the magnetization along the critical isotherm

$$
\begin{aligned}
& \chi / N=\left.\beta^{-1} \frac{\partial^{2} \ln Z / N}{\partial H^{2}}\right|_{\beta} \propto\left(T-T_{c}\right)^{-\gamma}, \\
& C_{H} / N=\left.k \beta^{2} \frac{\partial^{2} \ln Z / N}{\partial \beta^{2}}\right|_{H} \propto\left(T-T_{c}\right)^{-\alpha}, \\
& \left\langle\overrightarrow{\mathrm{S}}_{0} \cdot \overrightarrow{\mathrm{S}}_{r}\right\rangle \propto \begin{cases}\exp (-|\overrightarrow{\mathbf{r}}| / \xi), & T>T_{c}, \\
\frac{1}{r^{d-2+\eta}}, & T=T_{c} ;\end{cases}
\end{aligned}
$$




$$
\begin{gathered}
\xi \propto\left(T-T_{c}\right)^{-\nu} ; \\
\Re \propto H^{1 / \delta}, \quad T=T_{c} ; \\
\left(T-T_{c}\right)^{-2 \Delta} \propto \frac{\left.\frac{\partial^{2 n+2} \ln Z}{\partial H^{2 n+2}}\right|_{\beta}}{\left.\frac{\partial^{2 n} \ln Z}{\partial H^{2 n}}\right|_{\beta}}, \quad H=0 .
\end{gathered}
$$

In concert with the se definitions (5) and the scaling hypothesis (6), we may write (4) to display explicitly the dependence on inverse temperature $\beta$. Thus we have

$$
\ln Z / N \simeq \xi^{-2} Y\left(H \beta^{1 / 2} \xi^{2 / 2} \chi^{1 / 2}\right) .
$$

Form (7) insures the definition of $\chi$ and the scaling hypothesis (6) with

$$
\Delta=\frac{1}{2}(z \nu+\gamma+1),
$$

and is a form of weak scaling. ${ }^{5}$ When, in addition, we impose the (renormalization-group) assumption $z=d$, we have hyperscaling. The usual exponent relations $s^{6}$ then become

$$
\begin{aligned}
& \text { (i) } \gamma=1+(2-\eta) \nu, \\
& \text { (ii) } \delta=\Delta /(\Delta-\gamma), \\
& \text { (iii) } \Delta=\frac{1}{2}(d \nu+\gamma+1), \\
& \text { (iv) }-\alpha_{s}=d \nu,
\end{aligned}
$$

by form (7) and the definitions (5) and (6). The "singular-part" [relation (9) (iv) is equivalent to the limit formulation (1)] of the specific heat has in$\operatorname{dex} \alpha_{s}$, where it differs from $\alpha$. The modifications from the usual relations [only (9) (ii) remains the same] are due to the confluence of the singularity at $T=0$ with that at $T=T_{c}$ when $T_{c}=0$.

\section{SPHERICAL MODEL}

It is well known that the spherical model is the $n \rightarrow \infty$ limit of the classical $n$-component Heisenberg model $^{7}$ and that it can be treated in such a way that the space dimension $d$ plays the role of a continuous parameter. ${ }^{8}$ The critical temperature falls to zero as $d$ decreases to 2 . For $d>2$, the scaling form (4) is known to hold; however, many of the critical indices diverge ${ }^{9}$ as $d-2$. By use of the general formulas of Joyce, ${ }^{9}$ we have verified by direct calculation for $0<d<2$ the validity of form (7) with $z=d$. The critical indices for this model are

$$
\begin{array}{ll}
\alpha_{s}=-d /(2-d), & \gamma=2 /(2-d), \\
\nu=1 /(2-d), & \eta=2-d, \\
\Delta=2 /(2-d), & \delta=\infty,
\end{array}
$$

which satisfy the relations (9) and

$$
\ln Z / N \simeq \beta^{-d /(2-d)} Y\left[H \beta^{2 /(2-d)}\right] .
$$

The magnetization for $T=0$ is a constant in $H$ which gives [by (5)] the values of $\eta$ and $\delta$.

\section{IV. $n$-COMPONENT CLASSICAL HEISENBERG MODEL}

In the calculation of the properties of the $n$-component classical Heisenberg model of Stanley, ${ }^{7}$ we use the transfer-matrix method to compute the solution for the linear chain. The transfer matrix for this problem is

$$
T=\exp \left[\beta \vec{S}_{i}^{(n)} \circ \vec{S}_{i+1}^{(n)}\right],
$$

and its eigenvalues are ${ }^{10-12}$

$\lambda_{l}^{(n)}(\beta J)=(2 \pi)^{n / 2}(n \beta J)^{1-(n / 2)} I_{(n / 2)_{-1+l}}(n \beta J)$,

where $I_{\mu}$ is the modified Bessel function of the first kind of order $\mu$. From the analysis of Stanley, ${ }^{13}$ we have, under the normalization constraint $\overrightarrow{\mathrm{S}}^{2}$ $=n$ ),

$$
\begin{aligned}
& E / N=-n J y_{n}, \\
& \chi / N=\beta m^{2}\left(1+y_{n}\right) /\left(1-y_{n}\right), \\
& C_{H} / N=k(n \beta J)^{2}\left[1-y_{n}^{2}-(n-1) y_{n} /(n \beta J)\right], \\
& \left\langle\overrightarrow{\mathrm{S}}_{0} \circ \overrightarrow{\mathrm{S}}_{j}\right\rangle=n\left(y_{n}\right)^{j},
\end{aligned}
$$

where

$$
\begin{aligned}
y_{n} & \equiv \frac{\lambda_{1}^{(n)}(\beta J)}{\lambda_{0}^{(n)}(\beta J)}=\frac{I_{n / 2}(n \beta J)}{I_{(n / 2)-1}(n \beta J)} \\
& \sim 1-\frac{(n-1)}{2 n \beta J}+\frac{(n-1)(n-3)}{8(n \beta J)^{2}}+\cdots
\end{aligned}
$$

by the asymptotic expansion of the Bessel functions for large arguments. ${ }^{14}$ It is to be noted ${ }^{13}$ that when $n$ is 1 or 3 , the series (15) does not have the expected leading order terms, and, in fact, the correction terms to those explicitly given in (15) are of the form $e^{(-2 B J)}$. If we follow the analysis of McLean and Blume $\mathrm{e}^{15}$ for the four-spin correlation function, which generalizes directly from their case $n=3$ to general $n \geq 1$ in terms of the eigenvalues (13), we can compute, using

$$
z_{n} \equiv \frac{\lambda_{2}(\beta J)}{\lambda_{0}(\beta J)} \sim 1-1 / \beta J+\cdots,
$$

that

$$
\frac{\partial^{4} \ln Z / N}{\partial H^{4}} \propto \beta^{3}\left(\frac{K}{\left(1-y_{n}\right)^{2}\left(1-z_{n}\right)}+\frac{K^{\prime}}{\left(1-y_{n}\right)^{3}}\right),
$$

where $K$ and $K^{\prime}$ are constants. Thus by substitution of (15) and (16) into (14) and (17), we obtain

$$
\begin{array}{ll}
\gamma=2, & \alpha=0, \quad \alpha_{s}=-1 \\
\nu=1, & \eta=1, \quad \Delta=2, \quad \delta=\infty,
\end{array}
$$

for all $n$ except 1 and 3. (The definition of the magnetization in terms of the limit of the spin-spin correlations has been used.) Comparison with (10) shows that these results agree identically with those 
for the spherical model with $d=1$.

In the case $n=3$, the form

$$
C_{H} / N \simeq k(n-1)\left(\frac{1}{2}+c_{1} T+c_{2} T^{2}+\cdots\right)
$$

for specific heat fails, as all the $c_{i}$ coefficients vanish. The correct formula ${ }^{13,16}$ is

$$
C_{H}(n=3) / N=k\left[1-(\beta J)^{2} / \sinh ^{2}(\beta J)\right],
$$

which yields the anomalous value $\alpha_{s}=-\infty$.

In the case $n=1, \mathrm{Eq}$. (15) fails as every coefficient vanishes, and we get

$$
y_{1}=\tanh (\beta J) \simeq 1-2 e^{-2 \beta J} .
$$

This exponential approach causes, $\gamma, \nu$, and $\alpha$ to be infinite. [The coefficient $K$ in Eq. (17) vanishes for this case as well.] Even so, all the relations (9) continue to hold, with the exception of (9) (iv), if we interpret them as limits in the sense of Eq. (1). First we have $\eta=1$, since $y_{1}$ goes to unity as $T \rightarrow 0$. Then relation (9) (i) becomes

$$
\lim _{T \rightarrow 0}\left(\frac{\ln (\beta \xi N / \chi)}{-\ln T}\right)=0,
$$

which we directly verify as

$$
\chi / N \sim \beta m^{2} e^{2 \beta J}, \quad \xi \sim \frac{1}{2} e^{2 \beta J} .
$$

The explicit form for $\ln Z / N$ of Nelson and Fish$\mathrm{er}^{17}$ for this case

$$
\ln Z / N \simeq \beta J+e^{-2 \beta J}\left[1+(\beta m H)^{2} e^{4 \beta J}\right]^{1 / 2}
$$

with error terms of $e^{-2 \beta J}$ in comparison with the terms retained, allows us to observe that " $\beta$ " is replaced by $\beta e^{2 \beta J}$; so relation (9) (ii) correctly yields $\delta=\infty$. Relation (9) (iii) becomes

$$
\lim _{T \rightarrow 0} \frac{\ln \left(N \beta^{2} e^{4 \beta J} / \xi \chi \beta\right)}{-\ln T}=0,
$$

as expected. Since

$$
C_{H} / N=k(\beta J)^{2} \operatorname{sech}^{2}(\beta J),
$$

we see that relation (9) (iv) fails, i. e., Eq. (1). The reason is clearly that the derivative with respect to $\beta$ of terms like $e^{-2 \beta J}$ does not increase the divergence, as it does for a power of $\beta$.

Thompson ${ }^{18}$ has considered in detail the critical properties of the one-dimensional, spin- $\infty$ Ising model (see also Joyce ${ }^{19}$ ). For this model $\xi$ $\propto \beta^{-1} e^{2 \beta J}$, although Thompson did not compute the four-spin correlation functions, if we use the defi- nition $\Delta=2-\alpha-\gamma$, then relations (9) hold for this model! This result differs, of course, from the spin- $\frac{1}{2}$ Ising case where (9) (iv) fails.We would like to point out in connection with the $n$-vector models that the assertation of Balian and Toulouse ${ }^{12}$ that $\alpha=1$ for $n<1$ in the se models is somewhat artificial, as the coefficient of $\beta^{1}$ in $C_{H}$ exactly vanishes. This analytic continuation of the one-dimensional Stanley sequence is interesting in that $T_{c}>0$ for $n<1$. [Note that if $T_{c}$ continued to stick at zero, Eq. (19) would indicate a negative zero-point specific heat.] The scaling form for the logarithm of the partition function continues to hold, but $\alpha=0$ and a failure of the usual $\left(T_{c}>0\right)$ scaling law $d \nu=2-\alpha$ for the se models is the correct conclusion. It is to be noted that for $n=0$, the second- and third-largest eigenvalues are exactly degenerate for all temperatures, since for integral order $I_{m}=I_{-m}$, and so the critical behavior may not be given by the above analysis.

\section{SUMMARY}

In conclusion, we point out that for all the models considered here, the scaling form (7) holds with $z=d$ and relations (9) (i) $-(9)$ (iii) are valid. The $\alpha$ dependent relation (9) (iv) fails in a variety of cases for a number of reasons unrelated to the validity of the scaling form. For this class of models ( $n$ vector, $d$ dimensional, $d<4$ ) it appears, as is well known, that the only substantial evidence for the failure of hyperscaling as distinguished from the failure of a particular exponent relation, is the numerical evidence for the three-dimensional Ising model, where the best exponent values are $\mathrm{a}^{20-22}$

$$
\begin{aligned}
& \Delta=1.563 \pm 0.003, \gamma=1.250 \pm 0.003, \\
& \nu=0.638_{-0.001}^{+0.002},
\end{aligned}
$$

which imply by Eq. (8),

$$
z=2.94 \pm 0.02 \text {, }
$$

which shows a small but persistent difference from the value 3 , i.e., an anomalous dimension of the vacuum! The determination of an index $\Delta$ is, of course, a verification of the scaling form (7).

\section{ACKNOWLEDGMENTS}

The authors are happy to acknowledge several conversations on the subject of this paper with $M$. Blume and S. Krinsky.
*Work performed under the auspices of ERDA.

${ }^{1}$ For a recent review, see M. E. Fisher, Rev. Mod. Phys. 46, 597 (1974), Eq. (2.4).

${ }^{2}$ K. G. Wilson and J. Kogut, Phys. Rep。12C, 75 (1974).

${ }^{3} \mathrm{D}$. Ruelle, Statistical Mechanics (Benjamin, New York, 1969).

${ }^{4}$ M. G. Kendall, and A. Stuart, The Advanced Theory of Statistics (Hafner, New York, 1959), Vol. I.
${ }^{5}$ See G. Stell, Phys. Rev. B 8, 1271 (1973); B. Widom, J. Chem. Phys. 43, 3898 (1965); L. P. Kadanoff, Physics (N.Y.) 2, 263 (1966).

${ }^{6}$ M. E。 Fisher, Rep. Prog. Phys。, 30, 615 (1969), p. 717 .

${ }^{7}$ H. E. Stanley, Phys. Rev. 176, 718 (1968); see also

M. Kac and C. J. Thompson, Phys. Norv. 5, 163 (1971).

${ }^{8}$ P. R. Gerber and M. E. Fisher, Phys. Rev。B 10, 4697, 
(1974).

${ }^{9} \mathrm{G}$. S. Joyce, in Phase Transitions and Critical Phenomena, edited by C. Domb and M. S. Green (Academic, London, 1972), Vol. 2, p. 375.

${ }^{10} \mathrm{G}$. S. Joyce, Phys. Rev. 155, 478 (1967).

${ }^{11}$ H. E. Stanley, M. Blume, K. Matsuno, and S. Milosevic, J. Appl. Phys. 41, 1278 (1970).

${ }^{12} \mathrm{R}$. Balian and G. Toulouse, Ann. Phys. 83, 28 (1974).

${ }^{13}$ H. E. Stanley, Phys。Rev. 179, $570(1969)$.

${ }^{14} \mathrm{E}$. T. Whittaker and G。 N. Watson, A Course of Modern Analysis, 4th ed. (Cambridge U. P。, London, 1927), p. 373 .

${ }^{15}$ F. B. McLean and M. Blume, Phys. Rev. B 7, 1149
(1973).

${ }^{16}$ M. E. Fisher, Amer. J. Phys. 32, 343 (1964).

${ }^{17}$ D. R. Nelson and M. E. Fisher, Ann. Phys. 91, 226 (1975).

${ }^{18}$ C. J. Thompson, J. Math. Phys. 9 , 241 (1968).

${ }^{19}$ G. S. Joyce, Phys. Rev。 Lett. 19, 581 (1967)。

${ }^{20} \mathrm{M}$. A. Moore, D. Jasnow, and M. Wortis, Phys。Rev。 Lett。22, 940 (1969).

${ }^{21}$ J. W. Essam and D. L. Hunter, J. Phys. C 1, 392 (1968).

${ }^{22}$ D. S。 Gaunt and G. A. Baker, Jr., Phys。Rev。B 1 , 1184 (1970)。 